\title{
GASA4, a GA-stimulated gene, participates in light signaling in Arabidopsis
}

\author{
Ing-Chien Chen ${ }^{\mathrm{a}}$, Si-Chen Lee ${ }^{\mathrm{b}}$, Shu-Mei Pan ${ }^{\mathrm{a}}$, Hsu-Liang Hsieh ${ }^{\mathrm{a}, *}$ \\ ${ }^{a}$ Institute of Plant Biology, College of Life Science, National Taiwan University, Taiwan \\ ${ }^{\mathrm{b}}$ Department of Electrical Engineering, National Taiwan University, Taiwan
}

Received 12 February 2007; received in revised form 25 March 2007; accepted 27 March 2007

Available online 31 March 2007

\begin{abstract}
Gibberellic acid-stimulated transcripts in Arabidopsis (GASAs) were previously isolated as GA-stimulated transcript 1 from tomato (GAST1) homologs and induced by GA. Here, we report that GASA4 is differentially regulated by light and affected by photoreceptors and light signaling transducers in Arabidopsis. Interestingly, GASA4 was down-regulated by a far-red (FR) light signaling regulator far-red insensitive 219 (FIN219) under all light conditions. GASA4 T-DNA inserted mutant seedlings with undetectable GASA4 transcripts exhibited reduced sensitivity to red (R) and FR light, and can be rescued by the ectopic expression of GASA4; the gasa4 mutant also showed enhanced response to exogenous GA under the same conditions. Furthermore, the gasa4 mutant showed up-regulated expression of several key GA metabolism genes such as GA 20-oxidase 1 (GA20ox1), GA 3-oxidase 1 (GA3ox1) and GA 2-oxidase 2 (GA2ox2), and displayed increased expression of vital flowering time genes, including $G I, C O, F T$, and $S O C 1$, which leads to an early flowering phenotype under long-day conditions as compared to the wild type. Thus, GASA4, negatively regulated by FIN219, may play various roles to regulate hypocotyl elongation and flowering during Arabidopsis development. (C) 2007 Published by Elsevier Ireland Ltd.
\end{abstract}

Keywords: Gibberellin; GASA4; FIN219; Light signaling; Flowering

\section{Introduction}

Searching for gibberellic acid (GA)-responsive genes is a way to understand the GA-mediated signaling pathway. The gibberellic acid-stimulated in Arabidopsis (GASA) gene family was first reported by Herzog et al. [1], and five GASA members (GASA1-GASA5) showed a tissue-specific expression pattern. GASA1 and GASA4, rather than GASA2 and GASA3, were induced by GA. GASAl is expressed in siliques and flower buds and GASA4 in growing roots and flower buds, whereas GASA2 and GASA3 are exclusively expressed in dry siliques and seeds [1]. Furthermore, results of GUS promoter activity assay showed that GASA4 is primarily expressed in meristematic regions, which implies a role in cell division [2]. Related proteins were also found in genes of various plant species such as tomato GA-stimulated transcript 1 (GAST1) [3] and root

\footnotetext{
* Corresponding author at: No. 1, Sec. 4, Roosevelt Road, Taipei 106, Taiwan. Tel.: +886 23366 2540; fax: +886223918940.

E-mail address: hlhsieh@ntu.edu.tw (H.-L. Hsieh).
}

system-induced gene 1 (RSI-1) [4], the gene family GAinduced protein (Petunia GIP) [5], Gerbera hybrida homolog of GAST1 (Gerbera GEG) [6] and potato Snaking-1 and -2 in Solanum tuberosum (StSNI and 2) [7,8]. The main features of these related proteins are (1) a putative signal peptide of 18-23 residues; (2) a varying length of highly divergent amino acid residues right after the putative signal peptide in the $\mathrm{N}$ terminus; (3) a conservative cysteine-rich (12 cysteines) Cterminal domain; and (4) a small peptide of 88 to about 112 amino acids.

The functions of these proteins are not so related. Tomato GASTI expression is induced by GA and inhibited by ABA. Transgenic plants with overexpressed or reduced GASTI expression did not show any phenotype [9]. The Petunia GIP expression pattern coincides with corolla and stem cell elongation [10], but $G E G$ is expressed when cell elongation ceases [6]. As well, potato StSN2 is an antimicrobial peptide induced by wounding and pathogen infection, which suggests its involvement in plant defense systems [8]. These data suggest that these related proteins share a similar biochemical/ structural activity but are involved in different processes. 
Plant phenotypes are often the convergent results of interactions between environmental factors such as light and phytohormones. GA has been known to regulate various aspects of plant development, including embryogenesis, seed germination, stem elongation, flowering, tuber formation and fruit development [11,12]. Modulation of GA levels and the regulation of GA-mediated signaling components are important for plant developmental patterns. GA 20-oxidase (GA20ox) and GA3ox, involved in the last two steps of GA biosynthesis, as well as GA2ox, responsible for the deactivation of bioactive GA, are the key enzymes for GA levels in cells [13]. Especially, the expression of GA3oxl and GA3ox2, which catalyze the conversion of $\mathrm{GA}_{20}$ into bioactive $\mathrm{GA}_{1}$, is tightly regulated. Both AtGA3oxl and AtGA3ox2 mRNAs are present in the cortex and endodermis of embryo axes of germinating Arabidopsis seeds; however, only AtGA3oxl expression is under feedback control by the GA response pathway [14]. Recently, Curaba et al. [15] reported that AtGA3ox2 is negatively regulated during embryogenesis by $L E A F Y C O T Y$ LEDON2 (LEC2) and FUSCA3 (FUS3), which indicates that GA biosynthesis is down-regulated in plant embryos.

Although GASA4 is induced by GA and shown to have tissue-specific expression patterns, its physiological function in Arabidopsis remains unclear. Here, we describe our investigation of its involvement in light signaling in Arabidopsis, so implicated because GASA4 displays $62 \%$ homology with a mungbean gene, gibberellin- and infrared light-stimulated transcript 1 in Vigna radiate ( $V r G I R I)$, isolated from a study of infrared (IR) light-induced genes. Current results indicate that GASA4 is differentially regulated under various light conditions, particularly red (R) and far-red (FR) light, under which its T-DNA knock-out mutant gasa4 exhibits a long hypocotyl phenotype. Intriguingly, GASA4 was negatively regulated by a FR signaling component far-red insensitive 219 (FIN219) under all light conditions and was able to repress the expression of several key GA metabolism genes as well as a group of flowering-time genes from flower buds under long-day conditions. Furthermore, the gasa4 mutant also has an earlier flowering phenotype. Thus, GASA4, with a newly found function involved in light signaling, may play a vital role in crosstalk between light and GA signaling to regulate plant development such as hypocotyl elongation and flowering.

\section{Materials and methods}

\subsection{Plant material and light treatment}

Throughout this paper, the wild type is Arabidopsis thaliana ecotype Columbia. The T-DNA knock-out mutant gasa4 (SALK_042431) was obtained from the Arabidopsis Biological Resource Center (ABRC; Ohio State University, Columbus). All other mutants used in this study were described previously [16]. Surface sterilization and cold treatment of Arabidopsis seeds and seedling growth conditions were described previously [16]. The light sources were described previously [17], and light intensities for FR, R, blue and white light were 8.36, $21.24,3.47$ and $77 \mu \mathrm{mol} \mathrm{m}{ }^{-2} \mathrm{~s}^{-1}$, respectively.

\subsection{RNA extraction, RNA gel blot and RT-PCR analyses}

Total RNA was isolated from 4-day-old and different lighttreated Arabidopsis seedlings by the Eppie-tube RNA isolation method [18]. Twenty micrograms of total RNA was separated on $1.2 \%$ agarose-formaldehyde gel, and then transferred to the positively charged nylon membrane (Roche Diagnostics). DIGlabeled cDNA probes were synthesized by PCR. Hybridization and washing conditions were performed at $65^{\circ} \mathrm{C}$ with DIGlabeled cDNA probes following standard procedures according to the manufacturer's procedure (Roche Diagnostics). For all blots, equal loading was confirmed by ethidium bromide staining of ribosomal RNAs (25S and 18S). All RNA gel blot analyses were biologically and independently repeated twice for consistent results. Quantification of the mRNA levels was by use of the LAS-3000 FUJI image system (Fuji Photo Film Co. Ltd.). For RT-PCR analysis, RNA samples were treated with DNase I (Invitrogen) before reverse transcription. Primers used in this study were as follows: for ent-kaurene synthase $(K S)$ : sense, $5^{\prime}$-AAGGCTGCTCAAAGTTATCCACAT- $3^{\prime}$, and antisense, 5'-ATAAAGTTGCAGCCCCAGAGAAAT-3'; for entkaurenoic acid oxidase 1 (KAOI): sense, 5'-CTGACTCCTTCACTCGC- $3^{\prime}$, and antisense, 5'-CCTGAGACGCTTGTGTT$3^{\prime}$; for GA20oxl: sense, 5'-CTCATGAATACACGAGCC-3', and antisense, 5'-TGATACACCTTCCCAAATG-3'; for GA3oxl: sense, 5'-CGATTTCCGTAAACTTTGGC- $3^{\prime}$, and antisense, 5'-ATCCATTGGATAGGATGTGG-3' ${ }^{\prime}$; for GA2ox2: sense, 5' TTTCCGTGAGTCGGTGG- ${ }^{\prime}$, and antisense, $5^{\prime}$-CTCCGCCTCTTCCTCCG-3'; for GAI: sense, 5'-GTTGTGGCTTGTGATGGA- $3^{\prime}$, and antisense, 5'-GGATTTAGTTTGGCTTCG-3'; for RGA: sense, $5^{\prime}$-CTCTCCGATACTCTTCAGATG- $3^{\prime}$, and antisense, 5'-CAAGCCACCAGATTACAA- ${ }^{\prime}$; for GIGANTEA (GI): sense, 5'-GAGCTGTCTTTCTCCGTTGTTT- ${ }^{\prime}$, and antisense, 5'-CTTCAATAGATTGGATAAACCGTC-3'; for CONSTANS (CO): sense, $5^{\prime}$-AACAGTGACAGATCCAGAGAACAG- $3^{\prime}$, and antisense, $5^{\prime}$-TTCTCTGCATACGCTTTCCTTGAA-3'; for FLOWERING LOCUS T (FT): sense, 5'-CCTGCTACAACTGGAACAACCTTT-3' ${ }^{\prime}$, and antisense, $5^{\prime}$-GCTATATAGGCATCATCACCGTTCGTTACTCG-3'; f for SUPRESSOR OF OVEREXPRESSION OF CONSTANS 1 (SOCl): sense, 5'-GTTTCTGAAGAAAATATGCAGCATT$3^{\prime}$, and antisense, 5'-GAACAAGGTAACCCAATGAACAA- $3^{\prime}$.

\subsection{Genomic DNA isolation and Southern blot analysis}

Genomic DNA was isolated from rosette leaves of wild type and the gasa4 mutant grown under white light condition $(16 \mathrm{~h}$ light and $8 \mathrm{~h}$ dark) [18], digested with appropriate restriction enzymes, separated on $1 \%$ agarose gel, transferred to positive charged nylon membranes (Roche Diagnostics Corp., Mannhein, Germany), and hybridized at $65^{\circ} \mathrm{C}$ with GASA4 genomic DNA fragment labeled with digoxigenin-11-dUTP. The probe was amplified by PCR with GASA41 primer, 5'-TCTGGATCCATGGCTAAGTCATATGGAGCT- ${ }^{\prime}$ and GASA42 primer, 5' $^{\prime}$ TCTGAATTCAGGGCATTTTGGTCCACCCTC- $3^{\prime}$. The signal was detected by the chemiluminescent reaction with CSPD (disodium 3-(4-methoxyspiro(1,2-dioxetane-3,2'-(5'-chloro)tri- 
cycle [3.3.1.1]decan)-4-yl) phenyl phosphate) (Roche) according to manufacture's instruction.

\subsection{Complementation test of the gasa4 mutant}

To overexpress GASA4 in the gasa4 mutant, a BamHIEcoRI DNA fragment containing the full-length GASA4 cDNA was obtained by PCR amplification with the following primer pairs: sense, 5'-TCTGGATCCATGGCTAAGTCATATGGAGCT- $3^{\prime}$, and antisense, $5^{\prime}$-TCTGAATTCAGGGCATTTTGGTCCACCCTC-3' (underline indicates the built-in BamHI (GGATCC) and EcoRI (GAATTC) sites, respectively. The purified PCR product was ligated into the binary transformation vector pCAMBIA1390-cMyc. The resulting constructs were introduced into the Agrobacterium GV3101 by a standard method [19] and then transformed into the gasa4 mutant by floral dipping [20]. Transgenic plants containing transgenes were selected with $25 \mu \mathrm{g} / \mathrm{ml}$ hygromycin. Approximately 30 independent $\mathrm{T} 1$ transgenic plants were selected and grown to the T2 generation. Phenotypic analysis was carried out with at least 10 independent lines.

\subsection{Response of the gasa4 T-DNA inserted mutant to exogenous application of $G A$}

$\mathrm{GA}_{3}$ was added to agar plates containing MS-medium plus $0.3 \%$ sucrose to a final concentration of $50 \mu \mathrm{M}$. Equal amounts of solvent, $95 \%$ ethanol, were added as the control. Wild type and mutant seeds were sterilized and sown on the plates containing $50 \mu \mathrm{M} \mathrm{GA}$ as described previously [16]. Hypocotyl length of the seedlings grown for 4 days under FR and $\mathrm{R}$ light was measured as described previously [16].

\subsection{Determination of flowering time for the gasa4 T-DNA inserted line}

Wild-type Arabidopsis and homozygous T-DNA-inserted lines of gasa4 were grown under long-day $(16 \mathrm{~h}$ day and $8 \mathrm{~h}$ dark) conditions. Flowering time was recorded by use of two different indexes: the days from the seedlings sown to the soil to appearance of the first inflorescence and leaf number at bolting. More than 10 plants for each case were investigated.

\section{Results}

\subsection{Expression of GASA4 is differentially regulated by light}

GASA4 was previously shown to be induced by GA and developmentally regulated, and its expression among the members of the GASA gene family in Arabidopsis was the most abundant in young seedlings [1]. However, its physiological function in Arabidopsis remained to be elucidated. Moreover, GASA4 also shows $62 \%$ homology with a mungbean gene, $\operatorname{VrGIRI,~that~is~regulated~by~IR~and~FR~light~(data~not~shown),~}$ which implies its regulation by light. Thus, we found the level of GASA4 mRNA isolated from 4-day-old seedlings grown

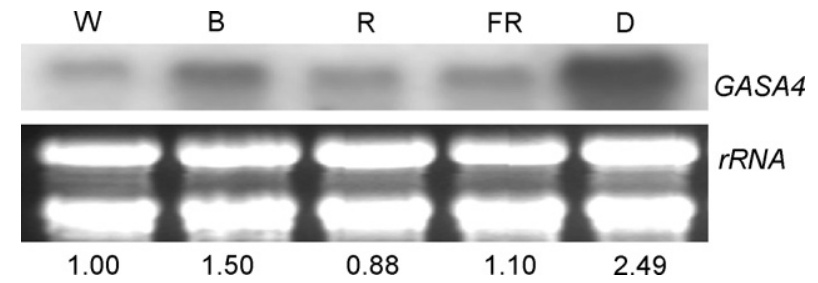

Fig. 1. RNA gel blot analysis of the expression of GASA4 under various light conditions. Total RNA was isolated from 4-day-old wild-type seedlings treated with different light, and $10 \mu \mathrm{g}$ total RNA was loaded for gel blot analysis. EtBrstained rRNA is shown below as a loading control. The numbers below the blot indicate normalized values based on the signal intensity in the samples and $25 \mathrm{~S}$ rRNA by using the LAS-3000 FUJI image system, with white light (W) set at 1 . B: blue light, R: red light, FR: far-red light, D: dark.

under different light conditions more than two-fold increased in darkness compared to white light and down-regulated in other light conditions, especially $\mathrm{R}$ and FR light (Fig. 1). So, GASA4 expression was differentially regulated in response to various quality of light.

\subsection{GASA4 is regulated by photoreceptors and light signaling transducers}

Since GASA4 was regulated by different light, we wondered whether it was involved in light signal transduction. RNA samples of various light signaling mutants, including photoreceptors grown under continuous FR light (cFR) for 4 days, were isolated and underwent RNA gel blot analysis.

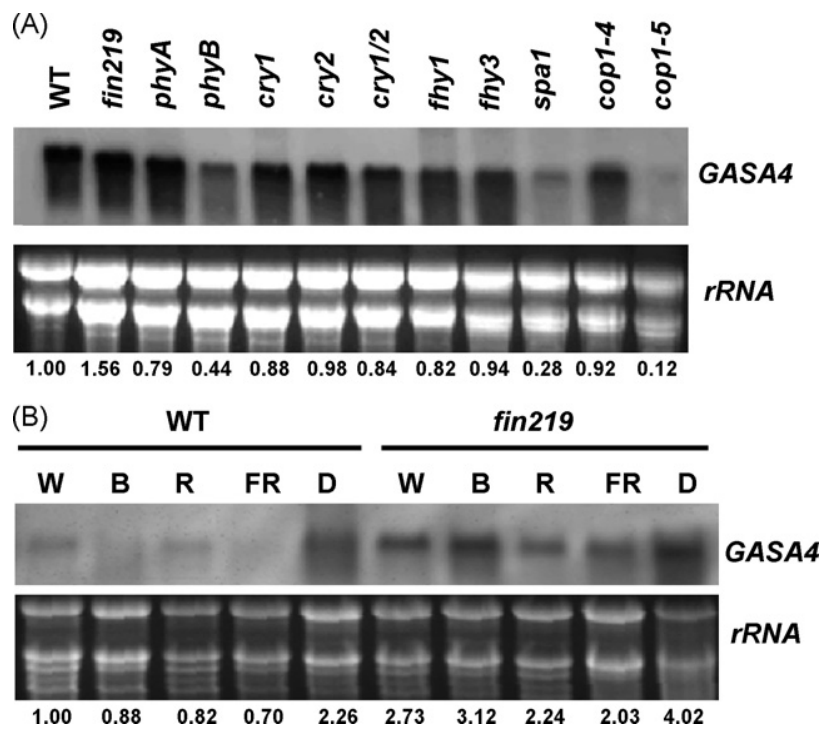

Fig. 2. RNA gel blot analysis of the expression of GASA4 in light signaling mutants under different light conditions. Total RNA isolated from the mutant seedlings of photoreceptors and light signaling components grown in continuous far-red light for 4 days (A), or continuous white (W), blue (B), red (R), and far-red (FR) light and dark (D) (B), was used for gel blot analysis of the expression of GASA4. Ethidium bromide-stained ribosomal RNA was shown as a loading control. The numbers below the blots indicate normalized values based on the signal intensity in the samples and 25S rRNA by using the LAS3000 FUJI image system, with WT in panel (A) or white light (W) for WT in panel (B) set at 1 . 
(A)

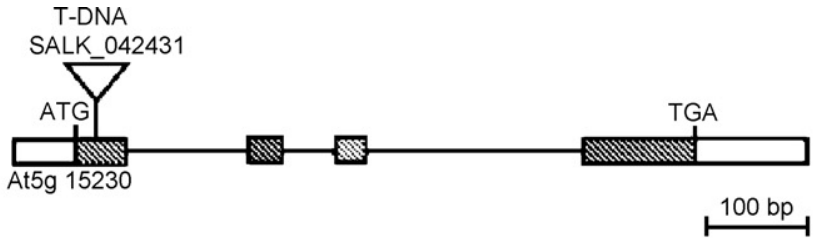

(B)

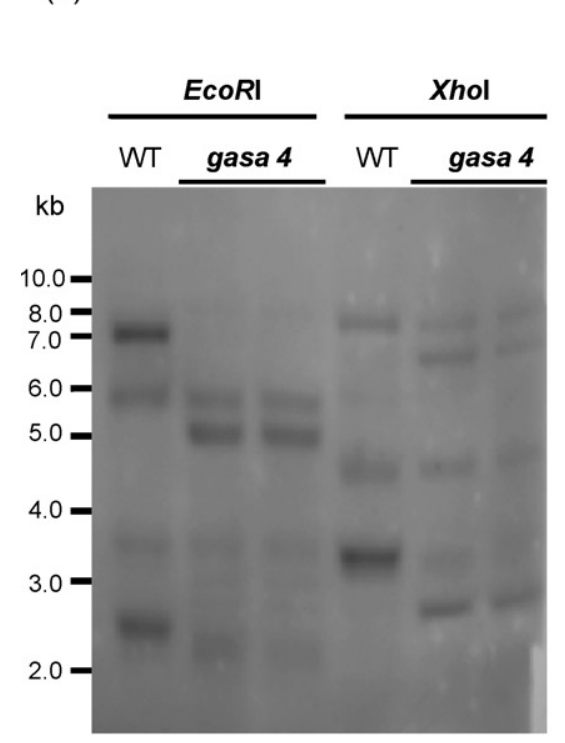

(C)

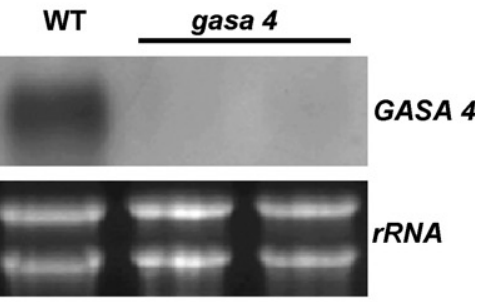

(D)

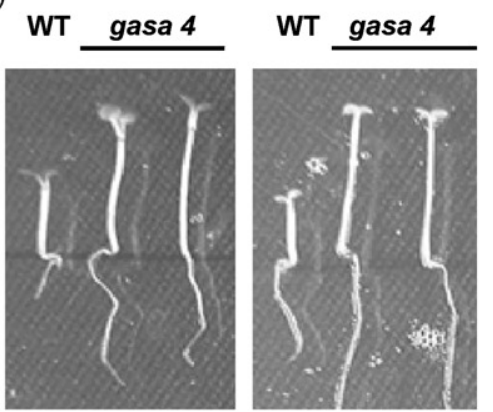

(E)

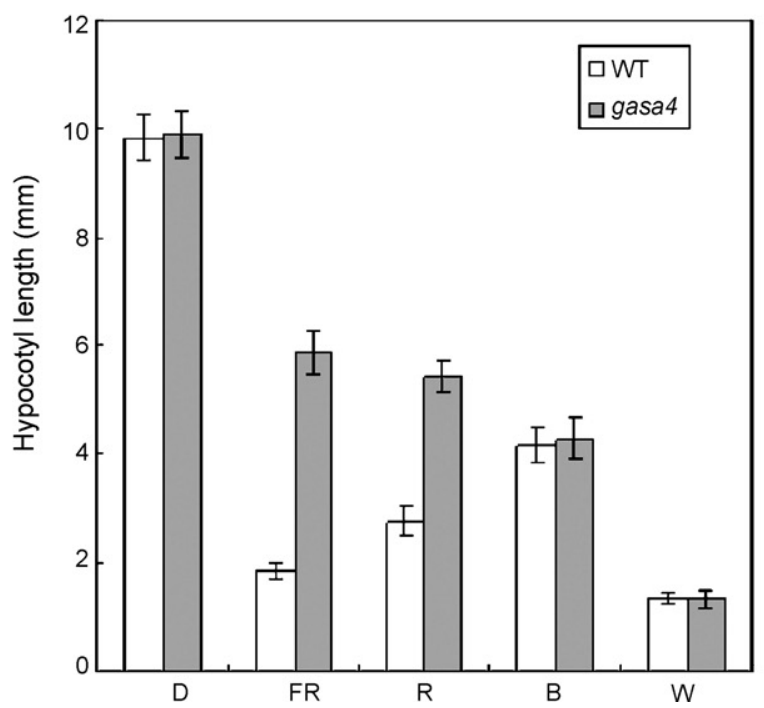

Fig. 3. Phenotypic investigation of the GASA4 T-DNA inserted mutant seedlings. (A) Schematic diagram of the GASA4 gene structure with location of T-DNA insertion. Boxes represent exons and thin lines are introns. The boxes with slash: translated regions, open boxes: untranslated regions. The line is in scale. (B) Genomic Southern analysis of the gasa4 T-DNA inserted mutant. Ten micrograms of genomic DNA isolated from wild type and the mutant seedlings grown in white light ( $16 \mathrm{~h}$ light, $8 \mathrm{~h}$ dark) were digested with restriction enzymes shown above the figure and subjected to genomic Southern analysis under stringent conditions. The probe used in the hybridization is a GASA4 genomic DNA fragment. WT: wild type, gasa4: gasa4 T-DNA inserted mutant. (C) RNA gel blot analysis of GASA4 expression in the gasa4 T-DNA inserted mutant seedlings grown in far-red light for 4 days. Ten micrograms total RNA was loaded for gel blot analysis. EtBr-stained rRNA is shown below as a loading control. Duplicated T-DNA inserted lines (gasa4) were used in the analysis. Phenotypic analysis of the seedlings of the gasa4 TDNA inserted lines grown under red light (D, left panel) and far-red light (D, right panel) conditions for 4 days. WT: wild type. (E) Quantitative measurement of the hypocotyl lengths of wild type and the gasa 4 mutant seedlings grown in various light conditions for 4 days. The number of the seedlings measured is $30(N=30)$. Error bars represent standard deviation. D: dark, FR: far-red, R: red light, B: blue light, W: white light. 
After normalization with loading control of rRNA levels, the expression of GASA4 was found to be substantially reduced in the mutants $p h y B$, suppressor of phytochrome A 1 (spal), constitutive photomorphogenic 1 (copl-5), a null allele of $C O P 1$, and slightly decreased in several photoreceptor mutants such as phyA and cryl as well as the fhyl mutant involved in FR signaling and the cop 1-4 mutant, a weak allele of COP 1 containing the N-terminal 282 amino acids of COP1 (Fig. 2A). In addition, since FIN219 has been shown to be a suppressor of COP1 and involved in the phyA-mediated signaling pathway [16], we further tested whether FIN219 was involved in the control of GASA4 expression. Surprisingly, GASA4 expression level was greatly increased about three-fold in the fin 219 mutant compared to the wild type
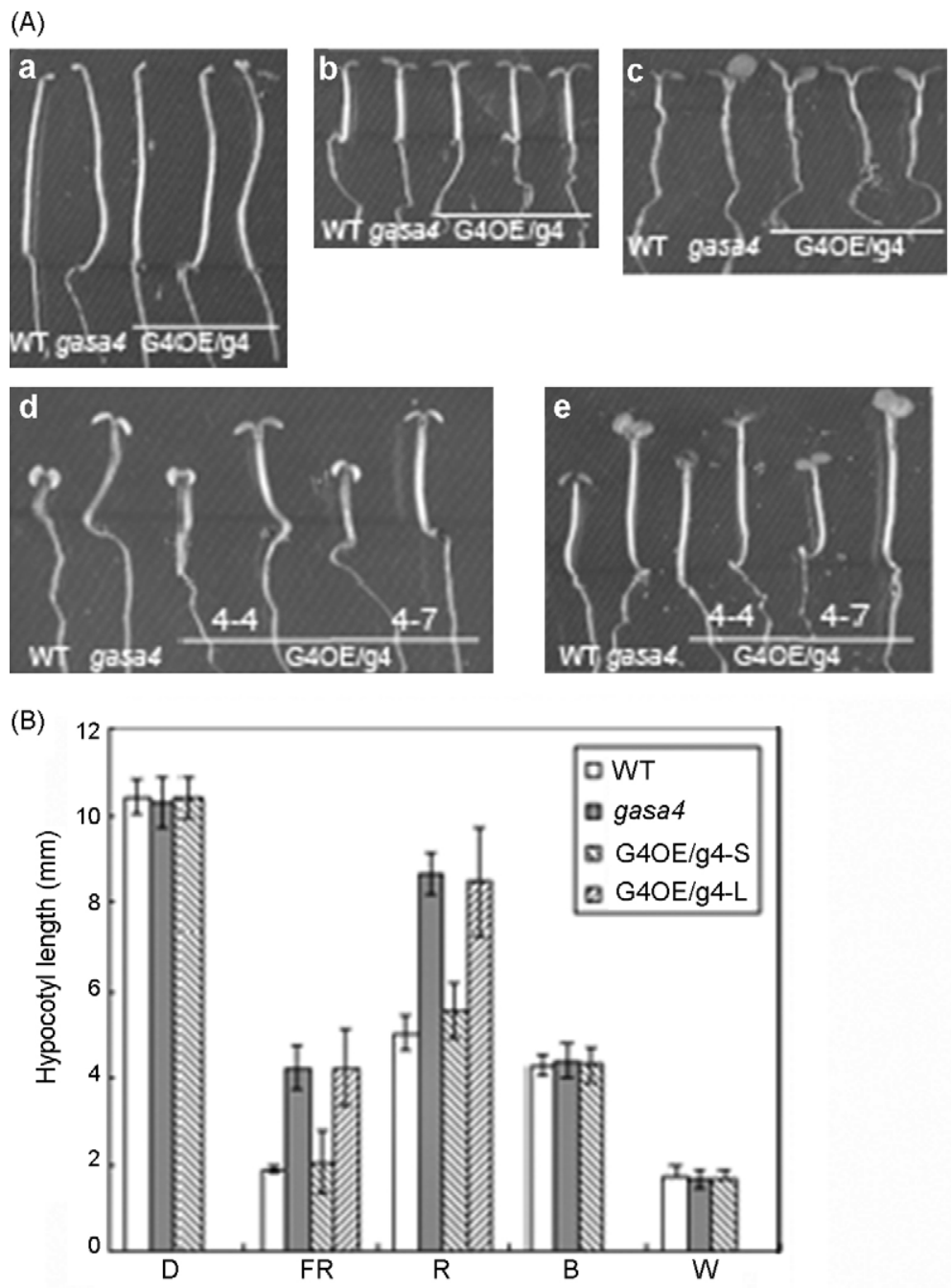

(C)

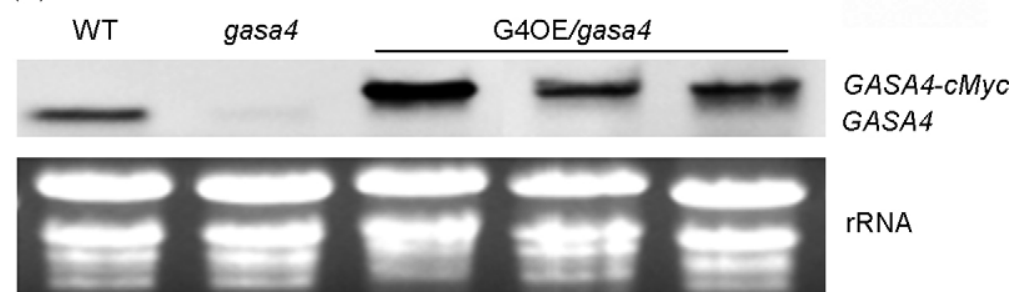

Fig. 4. Complementation of the gasa4 mutants. (A) Phenotypic examination of the gasa 4 transgenic seedlings harboring a $35 \mathrm{~S}$ promoter-driven cmyc-GASA4 fusion construct in the T2 generation grown in the dark (a), blue light (b), white light (c), far-red light (d), and red light (e) conditions for 4 days. 4-4 and 4-7 in (d) and (e) represent different transgenic lines. The segregated seedlings with long hypocotyls of the lines 4-4 and 4-7 shown in (d) and (e) are similar to the background mutant gasa4; the short seedlings are rescued phenotypes. (B) Quantitative measurement of the hypocotyl lengths of wild type and transgenic seedlings shown in (A). The number of the seedlings measured is $30(N=30)$. Error bars indicate standard deviation. G4OE/g4-S: GASA4 overexpressor/gasa4 mutant with short hypocotyls; G4OE/g4-L: GASA4 overexpressor/gasa4 mutant with long hypocotyls. (C) RNA gel blot analysis of GASA4 expression in transgenic seedlings. Total RNAs $10 \mu \mathrm{g}$ isolated from 4-day-old seedlings of three rescued transgenic lines grown under far-red light were loaded to each lane and subjected for RNA gel blot analysis. The probe is a Dig-labeled GASA4 cDNA. An ethidium-bromide stained gel in the bottom was shown for RNA equal loading. G4OE/gasa4: GASA4 overexpressor/gasa4 mutant. 
under all light conditions (Fig. 2B), which indicates that FIN219 negatively regulates GASA4 expression. Taken together, these data suggest that the GASA4 in Arabidopsis may act in a downstream manner and is regulated by light signaling components.

\subsection{The null mutant of GASA4 exhibits a hyposensitive phenotype under $R$ and FR light}

To further understand the function of GASA4 involved in light signaling, T-DNA insertion mutants were obtained from the Arabidopsis Biological Research Center (ABRC, Ohio) and underwent phenotypic examination under various light conditions. The mutant was derived from a T-DNA putatively inserted in the first exon of the GASA4 coding region that is composed of four exons and three introns (Fig. 3A). To further confirm whether the T-DNA was indeed inserted into the GASA4 locus, the genomic DNA isolated from wild type and the gasa4 mutant was digested with either EcoRI or XhoI and subjected to genomic Southern analysis with GASA4 gene as a probe. The result revealed that the genomic DNA digested with either EcoRI or XhoI did show polymorphism between wild type and the gasa4 mutant (Fig. 3B), indicating that the T-DNA was inserted in the GASA4 gene, leading to barely detected GASA4 transcripts in the gasa4 mutant (Fig. 3C). Moreover, homozygous 4-day-old seedlings of the gasa4 mutant examined under different light conditions displayed a hyposensitive phenotype, with long hypocotyls under continuous $\mathrm{R}$ light (cR) and cFR (Fig. 3D and E), respectively, but no phenotype under blue, white light and dark conditions (Fig. 3E), which implies that GASA4 plays a role in the control of hypocotyl elongation under both $\mathrm{R}$ and FR light.

\subsection{The null mutant of GASA4 can be rescued by ectopic expressions of GASA4}

To further understand whether GASA4 is mainly responsible for the phenotype of the gasa4 mutant shown under $\mathrm{cR}$ and $\mathrm{cFR}$, the GASA4 cDNA was driven by $35 \mathrm{~S}$ cauliflower mosaic virus promoter and introduced into the gasa4 mutant. The resulting transgenic lines were examined under various light conditions. Eleven out of 18 lines examined were found to have rescued phenotypes with short hypocotyls in T2 segregated seedlings under $\mathrm{cR}$ and $\mathrm{cFR}$, and two representative lines are shown here (Fig. 4A(d and e) and B); but they have no phenotype under other light conditions as well as in the dark (Fig. 4A(a-c) and B). To examine whether the rescued phenotype was due to the expression of the transgene GASA4, a RNA gel blot analysis of GASA4 in the rescued transgenic lines was carried out. It revealed that GASA4 was highly expressed in the rescued transgenic seedlings under $\mathrm{cFR}$ when compared to the genetic background gasa4 mutant (Fig. 4C), which indicates that GASA4 is indeed responsible for the mutant phenotype, involved in the control of hypocotyl elongation in response to $\mathrm{R}$ and FR light.
3.5. GASA4 represses several key enzyme-corresponding genes involved in GA metabolism

Although GASA4 was induced by GA [1], how it affects GAmediated responses remains unknown. To investigate whether GASA4 influences components involved in GA biosynthetic or signaling pathways, wild type and gasa4 mutant seedlings grown under cFR for 4 days underwent RT-PCR analysis of the 4 GA biosynthetic genes encoding the enzymes ent-kaurene synthase (KS), ent-kaurenoic acid oxidase 1 (KAOl), GA 3oxidase 1 (GA3oxl) and GA 20-oxidase 1 (GA20oxl); 1 GA deactivation enzyme, GA 2-oxidase 2 (GA2ox2) and 2 GAsignaling negative regulators, GA-insensitive $(G A I)$ and repressor of gal-3 (RGA). GA20ox1, GA3oxl and GA2ox2 mRNA levels were up-regulated in the seedlings of the gasa4 mutant, especially GA3oxl (Fig. 5). However, $K S$ and $K A O 1$, the early GA biosynthetic genes, and GAI and $R G A$, the GA signaling components, showed little change between the mutant and wild-type seedlings under cFR, which implies that GASA4 might modulate GA levels through negatively controlling the key enzymes-corresponding genes in GA metabolism.

\subsection{The gasa4 mutant exhibits increased response to exogenous $G A$}

The gasa4 mutant exhibited a hyposensitive phenotype under cFR (Fig. 3) and the expression of several key enzymecorresponding genes involved in GA metabolism was also

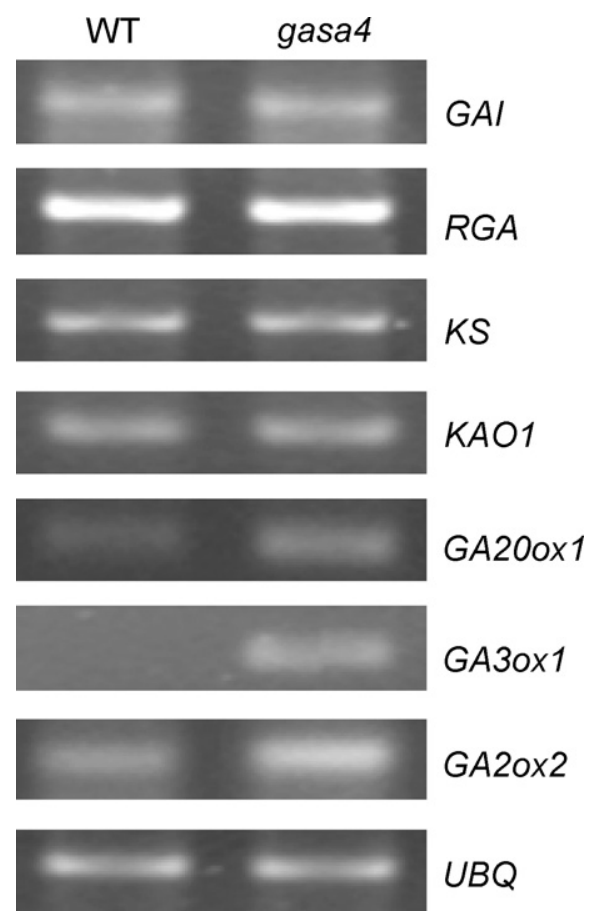

Fig. 5. RT-PCR analysis of the expression of GA biosynthetic and signaling genes in 4-day-old seedlings of the gasa4 mutant grown under far-red light. $U B Q$ : ubiquitin 10 as an endogenous loading control. WT: wild type, GAI: GAinsensitive, $R G A$ : repressor of gal-3, KS: ent-kaurene synthase, KAO1: entkaurenoic acid oxidase 1, GA20ox1: GA 20-oxidase 1, GA3ox1: GA 3-oxidase 1, GA2ox2: GA 2-oxidase 2. 


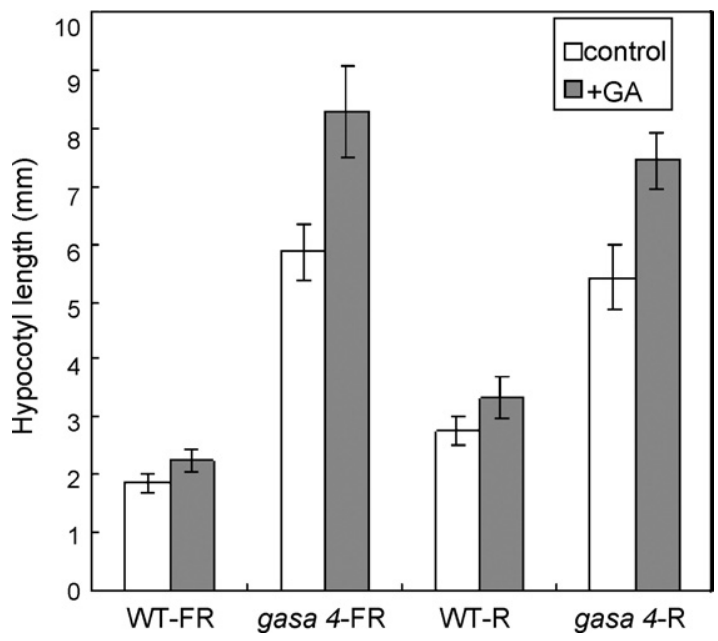

Fig. 6. Effect of exogenous application of gibberellins on hypocotyl phenotype of wild type and gasa 4 mutant under far-red and red light. Wild type (WT) and gasa 4 mutant seeds were sown on the GM plates with (+GA) or without (control) $50 \mu \mathrm{M}$ GA under far-red (FR) and red light (R) conditions for 4 days. $N=30$.

altered (Fig. 5). To further understand whether the long hypocotyl phenotype of the gasa4 mutant will alter its response to exogenous GAs, we tested the effects of exogenous GA on hypocotyl lengths of the gasa4 and wild-type seedlings grown under cFR and $\mathrm{cR}$. An amount of $50 \mu \mathrm{M} \mathrm{GA}_{3}$ could increase the hypocotyl length of wild-type seedlings by $20 \%$ as compared with the wild type without the addition of GA under both light conditions, whereas the hypocotyl length of the gasa4 mutant increased up to $41 \%$ and $37 \%$ in the presence of GA under cFR and cR, respectively, as compared to the gasa4 mutant without GA at the same conditions (Fig. 6), which indicates that the gasa 4 mutant increases response to GA. Thus far, the results suggest that GASA4 may affect GA metabolism and also modify responses to exogenous GA.

\subsection{The gasa4 mutant shows an earlier flowering phenotype under long-day conditions}

Since both light and GA play crucial roles in the regulation of flowering time in Arabidopsis, the expression and function of
GASA4 are associated with both factors, and GASA4 transcripts were detected in flower buds [1,2], we then asked whether GASA4 regulated flowering under long-day conditions by investigating the flowering time of the wild type and the gasa4 mutant. As compared to the wild type, the gasa 4 mutant showed an earlier flowering phenotype in terms of days to flowering and leaf number at bolting (Fig. 7A). To further explore how GASA4 altered flowering time, total RNA samples isolated from the floral buds of the wild type and gasa4 mutant grown under long-day conditions underwent RT-PCR analysis for several genes known to control flowering in the long-day pathway. All 4 genes analyzed, GIGANTEA (GI), CONSTANS (CO), FLOWERING LOCUS T (FT), and SUPRESSOR OF OVEREXPRESSION OF CO 1 (SOC1), especially GI, CO and $S O C 1$, were up-regulated in the gasa4 mutant (Fig. 7B), which is consistent with the earlier flowering phenotype of the gasa4 mutant shown in Fig. 7A. Thus, GASA4 can repress flowering by inhibiting key regulators of flowering in Arabidopsis under long-day conditions.

\section{Discussion}

GASA4, a member of the GASA gene family in Arabidopsis, was previously reported to be induced by GA. Here, we show that GASA4 function is associated with light-mediated inhibition of hypocotyl elongation and may modulate GA levels through controlling the expression of the genes for key enzymes in GA metabolism, as well as with control of the expression of major regulators of flowering time in Arabidopsis under long-day conditions. Therefore, GASA4 might play a crucial role to regulate hypocotyl elongation and flowering in response to the integration of light signaling and GAs.

At least 17 GASA members, forming a GASA gene family, are present in the Arabidopsis genome. GASA1-GASA4 were first reported to be induced by GA [1] and related to the tomato GAST1. GASA members have shown tissue-specific expression patterns, such as GASAl mainly in siliques and flower buds, GASA2 and GASA3 in dry seeds and GASA4 in flower buds $[1,2]$. These expression patterns suggest that the members might play roles in the development of reproductive tissues.

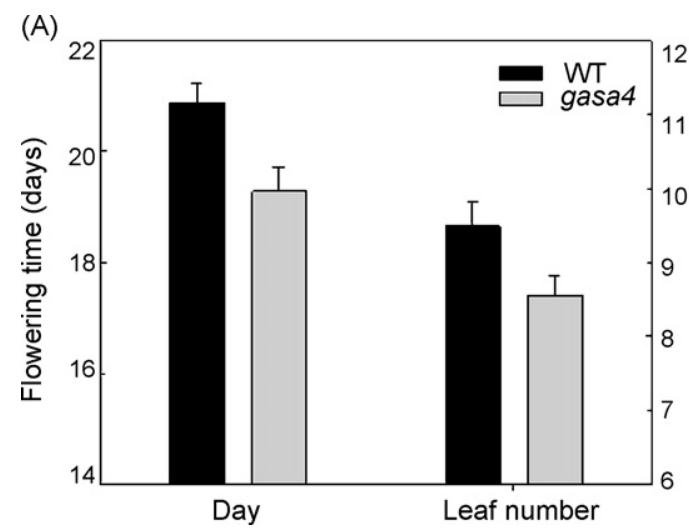

(B)

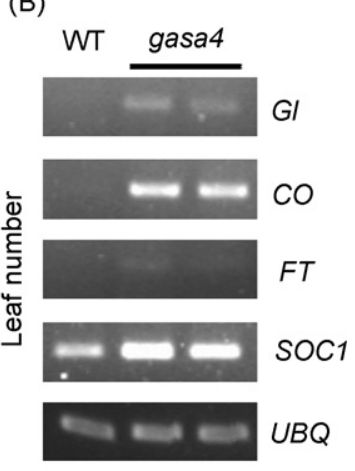

Fig. 7. Analysis of flowering time and the expression of several key flowering time genes in wild type (WT) and the gasa4 mutant. (A) Wild type and GASA4 T-DNA inserted mutant gasa4 were grown under long-day conditions ( $16 \mathrm{~h}$ day and $8 \mathrm{~h}$ night). Flowering time was recorded on the basis of the days from seedlings sown in soil to appearance of the first inflorescence and the leaf number at bolting. Ten plants for each case were investigated. (B) RT-PCR analysis of the expression of flowering-time genes in the flower buds of WT and the gasa4 mutant. UBQ, ubiquitin 10 as an endogenous loading control. 
Here, we found that GASA4 was regulated by different wavelengths of light (Fig. 1), which was further supported by multiple photoreceptors and light signaling components affecting GASA4 transcripts under cFR light (Fig. 2A). In particular, GASA4 transcripts were up-regulated in the fin219 mutant, a positive regulator involved in the phyA-mediated FR signaling pathway, under all light conditions (Fig. 2B), which implies that FIN219 can repress the expression of GASA4 in light signaling. GASA4 expression was highly reduced in the mutants of key modulators of light signaling such as COP1 and SPA1, which can regulate phyA activity [21-23]. Intriguingly, GASA4 transcripts were differentially expressed in the cop1-4 and cop1-5 alleles. Since the T-DNA-tagged cop1-5 is null [24] and has no COP1 protein, which resulted in barely detected GASA4 transcripts, whereas the copl-4 with only the Nterminal 282 amino acids caused a reduced expression of GASA4 transcripts, implying that COP1 may positively regulate GASA4 expression. However, COP1 has been shown to have E3 ligase activities that mediate protein degradation by $26 \mathrm{~S}$ proteasome [21-23]. Whether COP1 regulates GASA4 expression at the protein level remains to be elucidated. As well, the T-DNA knock-out mutant of GASA4 displayed a long hypocotyl phenotype under both $\mathrm{cR}$ and cFR light (Fig. 3) and ectopic expression of GASA4 can rescue the gasa4 mutant to the wild type like phenotype under the same conditions (Fig. 4). Thus, GASA4 is involved in the control of hypocotyl elongation in response to $\mathrm{R}$ and FR irradiation.

Furthermore, the photoreceptors phyB and cryl/cry 2 mutants grown under FR light showed reduced expression of the GASA4 transcripts (Fig. 2A), which suggests that the interactions between phyA and phyB (the photoreceptor for $\mathrm{R}$ perception) as well as cry1 and cry 2 (the photoreceptors for blue light perception) may be mainly responsible for the regulation of GASA4 expression. Since the control of hypocotyl elongation by interactions among various photoreceptors has been documented [25-27], the synergistic action for hypocotyl growth and cotyledon unfolding is conditional. Phytochrome A in the high-irradiance response mode can positively regulate phyB signaling [28], but phyA in the very-low-fluenceresponse mode negatively regulates phyB signaling [29]. So, phyB in a continuous FR condition positively affects GASA4 expression regulated by phyA (Fig. 2A). Similarly, cry1 and cry2 may work redundantly in FR to act synergistically with phyA to control GASA4 expression (Fig. 2A).

The early steps in the GA biosynthetic pathway are controlled by single-copy genes or small gene families. Two ent-kaurenoic acid oxidases (KAO1 and KAO2), ent-copalyl diphosphate synthase $(C P S)$ and ent-kaurene synthase $(K S)$ are encoded by single-copy genes in Arabidopsis [30]. By contrast, enzymes catalyzing the later reaction in this pathway are each encoded by a multiple gene family: GA20ox and GA3ox are encoded by four genes and GA2ox by six [30]. Regulations of the bioactive GA levels, whether under feedback or developmental regulation, are primarily determined by the enzymes in these three gene families. GA-mediated responses can be regulated through controlling specific biosynthetic genes. Recent studies provided evidence that the KNOX genes are involved in modulating GA levels in the SAM region by repressing the expression of GA20oxl [31]. Furthermore, the levels of $\mathrm{GA}_{1}$ and GA20ox mRNA in the KNOX-overexpressed plants were reduced as compared with that in the wild type. In addition, the embryonic regulators LEC2 and FUSCA (FUS3) are involved in multiple aspects of Arabidopsis seed development by inhibiting the expression of GA3ox2 to modulate GA levels [15]. In comparison, gene expression and enzyme activities are relatively steadier in CPS, KS, and KAOs [32]. Overexpressing either CPS or KS in Arabidopsis increases upstream gibberellin precursors but not bioactive GAs [33]. These findings are in accordance with our results, because GASA4 controlled the expression of only the later biosynthetic enzymes GA3ox1, GA20ox1 and GA2ox2 and did not alter $K S$ and $K A O 1$ expression (Fig. 5). These three later biosynthetic enzymes were used as the index in our study because they are the major enzymes expressed in each gene family at the Arabidopsis young seedling stage. All the three were upregulated when GASA4 is mutated (Fig. 5), so the gasa4 mutant may show a greater GA flux from GA precursors to active GAs and then to inactive catabolites.

In addition, during de-etiolation, the responses of plants to the endogenous pool of $\mathrm{GA}_{1}$ are reduced in continuous light compared with dark-grown plants [34]. The gasa4 mutant increased exogenous GA response under $\mathrm{R}$ and FR light (Fig. 6), which suggests that GASA4 negatively regulates GA signaling under de-etiolation condition. The gasa4 mutant may alter expression levels of the GA-responsive genes. Alternatively, the gasa4 mutant might increase sensitivities to exogenous GA, leading to even longer hypocotyls in the presence of GA under both FR and R conditions.

GAI and RGA are two of the five DELLA members controlling GA signaling in Arabidopsis. Their regulation is primary at the protein level through the GA-stimulated $26 \mathrm{~S}$ proteasome degradation pathway [35,36], so it is not surprising that gasa4 mutation did not alter RNA levels of GAI and RGA (Fig. 5). The interaction between light and GA-regulated hypocotyl elongation has been extensively investigated $[11,34,37]$. However, the relation between light and GA in photomorphogenesis has been a long-standing controversy; in particular, is phytochrome-mediated photomorphogenesis the net result of a reduction in GA content or GA sensitivity of the stem? De-etiolation of pea involves a rapid decrease of $\mathrm{GA}_{1}$ level to about $25 \%$ in apical shoots upon transfer to white light and an increase in $\mathrm{GA}_{1}$ content in plants transferred to the dark [37]. Recently, Alabadí et al. [38] reported that GA can repress photomorphogenesis in darkness, which indicates that both the chemical and genetic reduction of endogenous GA level derepresses photomorphogenesis in part in darkness. Thus, the GA-deficient gal-3 mutants display a much shorter hypocotyl phenotype than the wild type in darkness. In addition, some evidence also indicates that phytochromes regulate GA levels to control stem elongation and that photoperiod modulates GA content. For instance, GA levels decrease in PHYA-overexpressed tobacco plants and increase in antisensed PHYB transgenic potato; long-day treatment actually regulates two steps of GA biosynthesis $-\mathrm{GA}_{53}$ to $\mathrm{GA}_{44}$ and $\mathrm{GA}_{19}$ to 
$\mathrm{GA}_{20}$ - leading to lower $\mathrm{GA}_{19}$ and higher $\mathrm{GA}_{20}$ content upon transfer from short- to long-day conditions $[39,40]$. The latest evidence indicates that FR-rich long days or end-of-day FR conditions can stimulate petiole elongation by regulating the expression of GA20ox biosynthetic genes through PHYB-type PHYs and not PHYA [41]. However, the cross-talk modulator linking FR light and GA responses remains unclear. Here, we showed that the GASA4 in Arabidopsis can repress the GA biosynthetic genes GA3oxl, GA20oxl and GA2ox2 (Fig. 5). Whether GASA4 accordingly affects GA levels or GA signaling remains elusive. Furthermore, the gasa4 mutant exhibits a long hypocotyls phenotype and an earlier flowering, implying that endogenous active GA levels in the mutant may be higher than that in wild type. Our preliminary data reveal that the level of active $\mathrm{GA}_{4}$ in the gasa4 mutant grown under cFR is almost two-fold increase compared to wild type, which supports the long hypocotyl phenotype of gasa4 mutant observed under cFR. As well, FIN219 is gradually decreased under dark-FR transition (unpublished data), leading to GASA4 increase, thus reducing GA levels that result in photomorphogenic phenotype. This notion is consistent with the results shown in this study. In contrast, in the dark condition the photomorphogenic development will be repressed by a group of repressor proteins COP/DET/FUS [42]. How GASA4 and FIN219 are implicated in the repression of the photomorphogenic development in the dark remains to be answered. Thus, GASA4 acts as a vital crosstalk between FR signaling and GA metabolism under the control of FIN219 to regulate hypocotyl elongation and flowering.

GASA4 has been shown to display a tissue-specific expression pattern, with highly expressed in the meristematic regions [2]. Our result showed the gasa4 mutant caused increased expression of flowering time genes such as $G I, C O$, $F T$ and $S O C l$; leading to an early flowering phenotype (Fig. 7), which is consistent with previous reports that overexpression of flowering time genes resulted in early flowering phenotype $[43,44]$. This result also indicates that GASA4 functions as a negative regulator of Arabidopsis flowering under long-day condition, which is further supported by the fact that the fin 219 null mutant exhibits a delay flowering phenotype under both long- and short-day conditions (our unpublished data). Since FIN219, a positive component in phyA-mediated FR signaling pathway negatively regulates GASA4 expression (Fig. 2B), the GASA4 expression increases in the fin 219 null mutant, leading to reduced expression of flowering time genes as described in the above and a delay flowering phenotype. Therefore, a new finding is that GASA4 plays a role in light signaling and may function as a positive regulator of hypocotyl elongation of the seedlings as well as a negative regulator for the reproductive development of Arabidopsis.

\section{Acknowledgements}

This paper is dedicated to the memory of our colleague Prof. Shu-Mei Pan for her initiated research on infrared light. We thank Zhi-Gong Wang for reading and critically commenting on the manuscript, and the Arabidopsis Biological Resource
Center (ABRC; Ohio State University, Columbus) for providing T-DNA inserted mutant seeds. This work was supported by a grant from the National Science Council (NSC 91-2311-B002-045), Taiwan.

\section{References}

[1] M. Herzog, A.M. Dorne, F. Grellet, GASA, a gibberellin-regulated gene family from Arabidopsis thaliana related to the tomato GAST1 gene, Plant Mol. Biol. 27 (1995) 743-752.

[2] D. Aubert, M. Chevillard, A.M. Dorne, G. Arlaud, M. Herzog, Expression patterns of GASA genes in Arabidopsis thaliana: the GASA4 gene is upregulated by gibberellins in meristematic regions, Plant Mol. Biol. 36 (1998) 871-883.

[3] L. Shi, R.T. Gast, M. Gopalraj, N.E. Olszewski, Characterization of a shoot-specific, $\mathrm{GA}_{3}$ - and ABA-regulated gene from tomato, Plant J. 2 (1992) 153-159.

[4] B.H. Taylor, C.F. Scheuring, A molecular marker for lateral root initiation: the RSI-1 gene of tomato (Lycopersicon esculentum Mill) is activated in early lateral root primordial, Mol. Gen. Genet. 243 (1994) 148-157.

[5] G. Ben-Nissan, D. Weiss, The petunia homologue of tomato gast1: transcript accumulation coincides with gibberellin-induced corolla cell elongation, Plant Mol. Biol. 32 (1996) 1067-1074.

[6] M. Kotilainen, Y. Helariutta, M. Mehto, E. Pollanen, V.A. Albert, P. Elomaa, T.H. Teeri, GEG participates in the regulation of cell and organ shape during corolla and carpel development in Gerbera hybrida, Plant Cell 11 (1999) 1093-1104.

[7] A. Segura, M. Moreno, F. Madueno, A. Molina, F. Garcia-Olmedo, Snakin-1, a peptide from potato that is active against plant pathogens, Mol. Plant-Microbe Interact. 12 (1999) 16-23.

[8] M. Berrocal-Lobo, A. Segura, M. Moreno, G. Lopez, F. Garcia-Olmedo, A. Molina, Snakin-2, an antimicrobial peptide from potato whose gene is locally induced by wounding and responds to pathogen infection, Plant Physiol. 128 (2002) 951-961.

[9] L. Shi, N.E. Olszewski, Gibberellin and abscisic acid regulate GAST1 expression at the level of transcription, Plant Mol. Biol. 38 (1998) 10531060.

[10] G. Ben-Nissan, J.Y. Lee, A. Borohov, D. Weiss, GIP, a Petunia hybrida GA-induced cysteine-rich protein: a possible role in shoot elongation and transition to flowering, Plant J. 37 (2004) 229-238.

[11] J.L. Garcia-Martinez, J. Gil, Light regulation of gibberellin biosynthesis and mode of action, J. Plant Growth Regul. 20 (2002) 354-368.

[12] C.M. Fleet, T.P. Sun, A DELLAcate balance: the role of gibberellin in plant morphogenesis, Curr. Opin. Plant Biol. 8 (2005) 77-85.

[13] N. Olszewski, T.P. Sun, F. Gubler, Gibberellin signaling: biosynthesis, catabolism, and response pathways, Plant Cell 14 (Suppl) (2002) S61S80.

[14] S. Yamaguchi, Y. Kamiya, T.P. Sun, Distinct cell-specific expression patterns of early and late gibberellin biosynthetic genes during Arabidopsis seed germination, Plant J. 28 (2001) 443-453.

[15] J. Curaba, T. Moritz, R. Blervaque, F. Parcy, V. Raz, M. Herzog, G. Vachon, AtGA3ox2, a key gene responsible for bioactive gibberellin biosynthesis, is regulated during embryogenesis by LEAFY COTYLEDON2 and FUSCA3 in Arabidopsis, Plant Physiol. 136 (2004) 36603669.

[16] H.L. Hsieh, H. Okamoto, M. Wang, L.H. Ang, M. Matsui, H. Goodman, X.W. Deng, FIN219, an auxin-regulated gene, defines a link between phytochrome A and the downstream regulator COP1 in light control of Arabidopsis development, Genes Dev. 14 (2000) 1958-1970.

[17] J.L. Peters, M. Széll, R.E. Kendrick, The expression of light-regulated genes in the high-pigment-1 mutant of tomato, Plant Physiol. 117 (1998) 797-807.

[18] H.L. Hsieh, C.G. Tong, C. Thomas, S.J. Roux, Light-modulated abundance of an mRNA encoding a calmodulin-regulated, chromatin-associated NTPase in pea, Plant Mol. Biol. 30 (1996) 135-147.

[19] J. Sambrook, D.W. Russell, Molecular Cloning: A Laboratory Manual, 3rd ed., Cold Spring Harbor Laboratory Press, Cold Spring Harbor, 2001 
[20] S.J. Clough, A.F. Bent, Floral dip, a simplified method for Agrobacteriummediated transformation of Arabidopsis thaliana, Plant J. 16 (1998) 735743.

[21] Y. Saijo, J.A. Sullivan, H. Wang, J. Yang, Y. Shen, V. Rubio, L. Ma, U. Hoecker, X.W. Deng, The COP1-SPA1 interaction defines a critical step in phytochrome A-mediated regulation of HY5 activity, Genes Dev. 17 (2003) 2642-2647.

[22] H.S. Seo, J.Y. Yang, M. Ishikawa, C. Bolle, M. Ballesteros, N.H. Chua, LAF1 ubiquitination by COP1 controls photomorphogenesis and is stimulated by SPA1, Nature 423 (2003) 995-999.

[23] H.S. Seo, E. Watanabe, S. Tokutomi, A. Nagatani, N.H. Chua, Photoreceptor ubiquitination by COP1 E3 ligase desensitizes phytochromes A signaling, Genes Dev. 18 (2004) 1-6.

[24] T.W. McNellis, A.G. von Arnim, T. Araki, Y. Komeda, S. Misera, X.-W. Deng, Genetic and molecular analysis of an allelic series of cop1 mutants suggests functional roles for multiple protein domains, Plant Cell 6 (1994) 487-500.

[25] L. Hennig, C. Poppe, S. Unger, E. Schäfer, Control of hypocotyl elongation in Arabidopsis thaliana by photoreceptor interaction, Planta 208 (1999) 257-263.

[26] J.L. Weller, N. Beauchamp, L.H.J. Kerckhoffs, J.D. Platten, J.B. Reid, Interaction of phytochromes A and B in the control of de-etiolation and flowering in pea, Plant J. 26 (2001) 283-294.

[27] M.A. Mazzella, J.J. Casal, Interactive signaling by phytochromes and cryptochromes generates de-etiolation homeostasis in Arabidopsis thaliana, Plant Cell Environ. 24 (2001) 155-161.

[28] J.J. Casal, Coupling of phytochromes B to the control of hypocotyl growth in Arabidopsis, Planta 196 (1995) 23-29.

[29] J.J. Casal, H. Boccalandro, Co-action between phytochrome B and HY4 in Arabidopsis thaliana, Planta 197 (1995) 213-218.

[30] P. Hedden, A.L. Phillips, Gibberellin metabolism: new insights revealed by the genes, Trends Plant Sci. 5 (2000) 523-530.

[31] A. Hay, H. Kaur, A. Phillips, P. Hedden, S. Hake, M. Tsiantis, The gibberellin pathway mediates KNOTTED1-type homeobox function in plants with different body plans, Curr. Biol. 12 (2002) 1557-1565.

[32] S.E. Davidson, S.M. Swain, J.B. Reid, Regulation of the early GA biosynthesis pathway in pea, Planta 222 (2005) 1010-1019.
[33] C.M. Fleet, S. Yamaguchi, A. Hanada, H. Kawaide, C.J. David, Y. Kamiya, T.P. Sun, Overexpression of AtCPS and AtKS in Arabidopsis confers increased ent-kaurene production but no increase in bioactive gibberellins, Plant Physiol. 132 (2003) 830-839.

[34] D.P. O'Neill, J.J. Ross, J.B. Reid, Changes in gibberellin $A_{1}$ levels and response during de-etiolation of pea seedlings, Plant Physiol. 124 (2000) 805-812.

[35] A. Dill, S.G. Thomas, J. Hu, C.M. Steber, T.P. Sun, The Arabidopsis F-box protein SLEEPY1 targets gibberellin signaling repressors for gibberellininduced degradation, Plant Cell 16 (2004) 1392-1405.

[36] L. Tyler, S.G. Thomas, J. Hu, A. Dill, J.M. Alonso, J.R. Ecker, T.P. Sun, Della proteins and gibberellin-regulated seed germination and floral development in Arabidopsis, Plant Physiol. 135 (2004) 1008-1019.

[37] J. Gil, J.L. García-Martinez, Light regulation of gibberellin A1 content and expression of genes coding for GA 20-oxidase and GA $3 \beta$-hydroxylase in etiolated pea seedlings, Physiol. Plant. 108 (2000) 223-229.

[38] D. Alabadí, J. Gil, M.A. Blázquez, J.L. Garcia-Martinez, Gibberellins repress photomorphogenesis in darkness, Plant Physiol. 134 (2004) 10501057.

[39] J.D. Metzger, J.A.D. Zeevaart, Comparison of the levels of six endogenous gibberellins in roots and shoots of spinach in relation to photoperiod, Plant Physiol. 66 (1980) 679-683.

[40] S.J. Gilmour, J.A.D. Zeevaart, L. Schwenen, J.E. Graebe, Gibberellin metabolism in cell-free extracts from spinach leaves in relation to photoperiod, Plant Physiol. 82 (1986) 190-195.

[41] T. Hisamatsu, R.W. King, C.A. Helliwell, M. Koshioka, The involvement of gibberellin 20-oxidase genes in phytochromes-regulated petiole elongation of Arabidopsis, Plant Physiol. 138 (2005) 1106-1116.

[42] N. Wei, X.-W. Deng, Making sense of the COP9 signalosome. A regulatory protein complex conserved from Arabidopsis to human, Trends Genet. 15 (1999) 98-103.

[43] J. Putterill, F. Robson, K. Lee, R. Simon, G. Coupland, The CONSTANS gene of Arabidopsis promotes flowering and encodes a protein showing similarities to zinc finger transcription factors, Cell 80 (1995) 847-857.

[44] Y. Komeda, Genetic regulation of time to flower in Arabidopsis thaliana, Annu. Rev. Plant Biol. 55 (2004) 521-535. 